\title{
Case of a Superficial Hypopharyngeal Cancer at the Pharyngoesophageal Junction Which Is Detected by Transnasal Endoscopy Using Trumpet Maneuver
}

\author{
Kenro Kawada1, Tatsuyuki Kawano1, Taro Sugimoto², Toshihiro Matsui', \\ Masafumi Okuda1, Taichi Ogo', Yuuichiro Kume', Yutaka Nakajima1, \\ Katsumasa Saito1, Naoto Fujiwara1, Tairo Ryotokuji1, Yutaka Miyawaki', \\ Yutaka Tokairin', Yasuaki Nakajima1, Kagami Nagai' ${ }^{1}$, Takashi Ito ${ }^{3}$ \\ ${ }^{1}$ Department of Esophageal and General Surgery, Tokyo Medical and Dental University, Tokyo, Japan \\ ${ }^{2}$ Department of Otorhinolaryngology, Tokyo Medical and Dental University, Tokyo, Japan \\ ${ }^{3}$ Department of Human Pathology, Tokyo Medical and Dental University, Tokyo, Japan \\ Email: kawada.srg1@tmd.ac.jp, kawano.srg1@tmd.ac.jp, tarooto@tmd.ac.jp, toshihiro.911@gmail.com, \\ okuda.srg1@tmd.ac.jp, ogotaichi1105@yahoo.co.jp, yuukun@yg8.so-net.ne.jp, ddabohaze8341@yahoo.co.jp, \\ k-saito.srg1@tmd.ac.jp, fujiwara.srg1@tmd.ac.jp, ryotokuji.srg1@tmd.ac.jp, miyawaki.srg1@tmd.ac.jp, \\ tokairin.srg1@tmd.ac.jp, yasu.nakajima.srg1@tmd.ac.jp, nagai.srg1@tmd.ac.jp, t.ito.pth1@tmd.ac.jp
}

Received 10 November 2014; revised 17 December 2014; accepted 29 December 2014

Copyright (C) 2015 by authors and Scientific Research Publishing Inc. This work is licensed under the Creative Commons Attribution International License (CC BY). http://creativecommons.org/licenses/by/4.0/

\section{(c) (i) Open Access}

\begin{abstract}
Aims: In order to observe the pharyngoesophageal junction, the trumpet maneuver with transnasal endoscopy was used. Its efficacy is reported here. Material and Methods: A 71-year-old man who underwent esophagectomy in esophageal cancer 2 years ago was admitted to our hospital with primary pharyngeal cancer. The type of upper endoscope used was EG-580NW (FUJI Film, Tokyo, Japan). The endoscope is a trans-nasal endoscope that can provide high quality endoscopic images. The pharyngeal cancer located at right lateral wall which was previously detected could be observed easily. We asked the patient to blow hard and puff his cheeks with his mouth closed. When inspecting the hypopharynx and the orifice of the esophagus, we told the patient that we would start the trumpet maneuver. Results: After the trumpet maneuver, the pharyngeal mucosa was stretched out. Another primary lesion was observed at the posterior wall of the hypopharynx near the pharyngoesophageal junction. This technique provided a much better view of the lesion than had been possible with trans-oral endoscopy, allowing us to remove the biopsy specimen without sedation. Conclusion: In conclusion, the trumpet maneuver using trans-nasal endoscopy is a useful technique for precise inspection before treatments for patients who have already detected the lesions.
\end{abstract}




\section{Keywords}

\section{Superficial Pharyngeal Cancer, Transnasal Endoscopy, Trumpet Maneuver}

\section{Introduction}

The more progress achieved in endoscopy, the more superficial cancers in the head and neck regions have been found. There are, however, some areas difficult to observe with trans-oral endoscopy. The circumferential observation of hypopharyngeal mucosa is difficult during conventional endoscopy because of its anatomically closed nature, pharyngeal reflex and salivary accumulation. Recently, we applied new trans-nasal esophagogastroduodenoscopy (EGD) (EG-580NW, Fuji Film, Tokyo, Japan) with FICE system and modifications of endoscopic techniques for observing head and neck cancers and obtaining excellent results. The endoscope is a trans-nasal endoscope that can provide high quality endoscopic images to be viewed on a monitor and digitally recorded with a wide field view of 140 degrees. We herein report a case of superficial hypopharyngeal cancer which is detected by trans-nasal endoscopy with trumpet maneuver.

\section{Case Presentation}

A 71-year-old man who underwent esophagectomy in esophageal cancer 2 years ago was admitted to our hospital with primary pharyngeal cancer.

Before treatment, the upper gastrointestinal screening was studied by trans-nasal endoscopy (model EG-580NW, FUJI Film, Tokyo, Japan). The patient was asked to bow their head deeply in the left lateral position, and then we put our hand on the back of the patient's head and pushed it forward by one span of our hand. Then, he was asked to lift up their chin as far as possible. After the local anesthesia of the nose without sedation, the endoscope was inserted through the nose. The pharyngeal cancer located at right lateral wall which was previously detected could be observed easily (Figure 1). When inspecting the hypopharynx and the orifice of the esophagus, we told the patient that we would start the trumpet maneuver. We asked the patient to blow hard and puff his cheeks with his mouth closed (Figure 2). After the trumpet maneuver, the pharyngeal mucosa was stretched out (Figure 3). Another primary lesion was observed at the posterior wall of the hypopharynx near the pharyngoesophageal junction (Figure 4). This provided a much better view of the lesion than had been possible with trans-oral endoscopy. We could got the biopsy specimen without sedation (Video: http://blog.livedoor.jp/kenzaburou4641/). A $10 \times 7 \times 4 \mathrm{~mm}$ superficial lesion was removed by en bloc resection (Figure 5). Histopathological examination revealed a diagnosis of invasive squa- mous cell carcinoma (Figure 6).

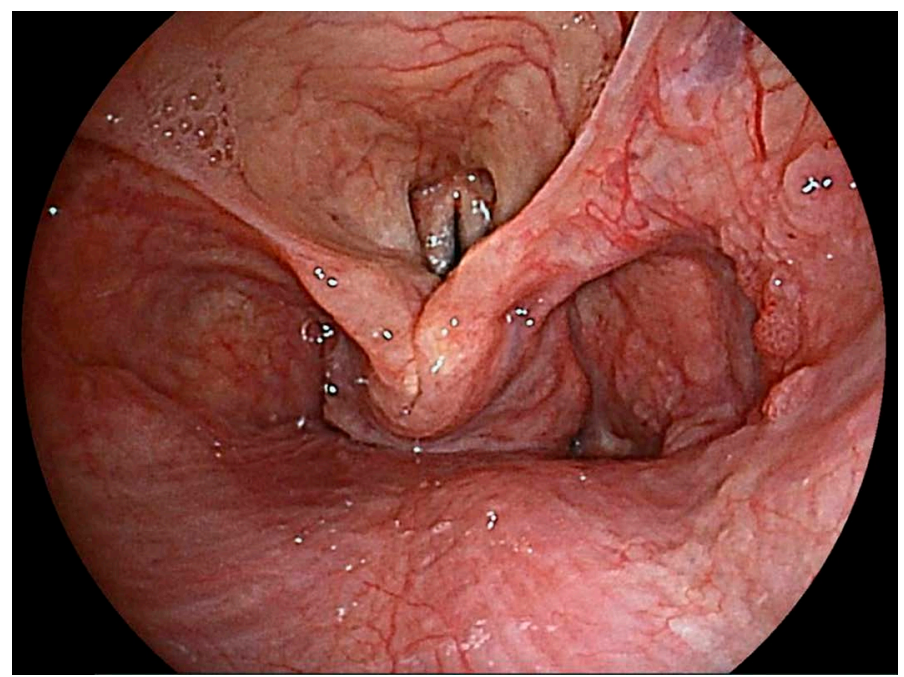

Figure 1. The endoscopic view of the primary pharyngeal cancer. There was no another cancer under the normal study. 


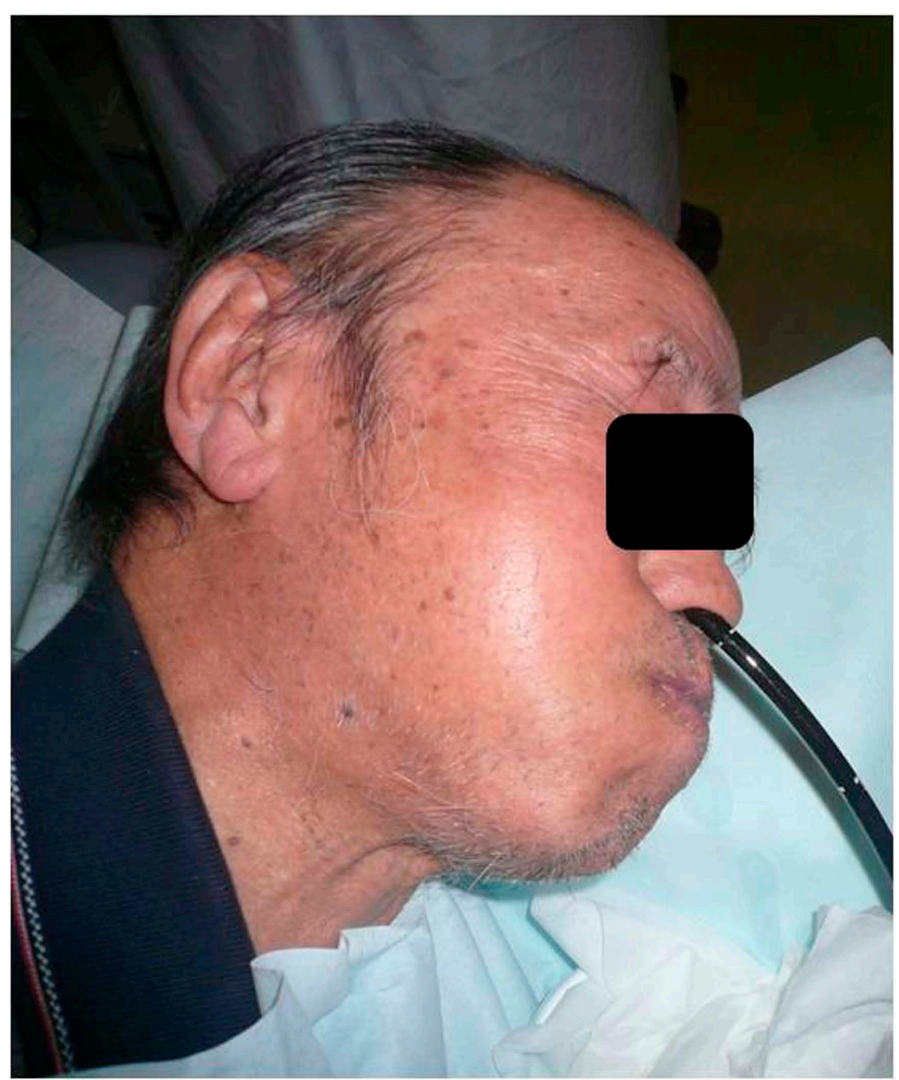

Figure 2. The patient is asked to blow hard and puff their cheeks while the mouth remains closed.

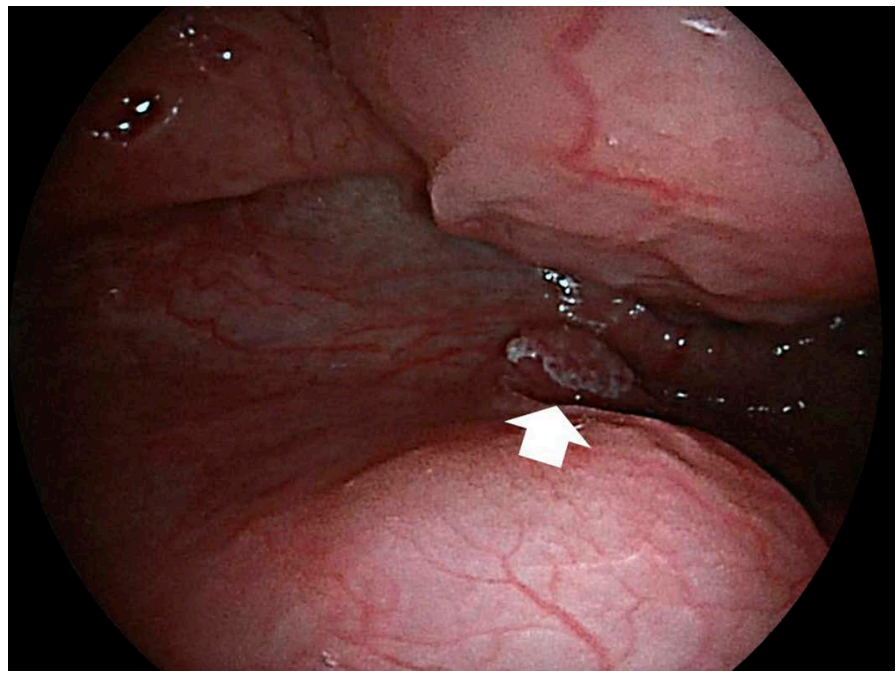

Figure 3. The pharyngeal mucosa was stretched out; tumor was observed (arrows).

\section{Discussion}

Head and neck cancers, especially pharyngeal cancer, frequently co-exist with esophageal cancer, but pharyngeal cancer has proven difficult to detect before its progression to an advanced stage [1]. Recent progress in endoscopic technologies has allowed narrow-band imaging (NBI) of the pharyngeal regions and has eliminated the 


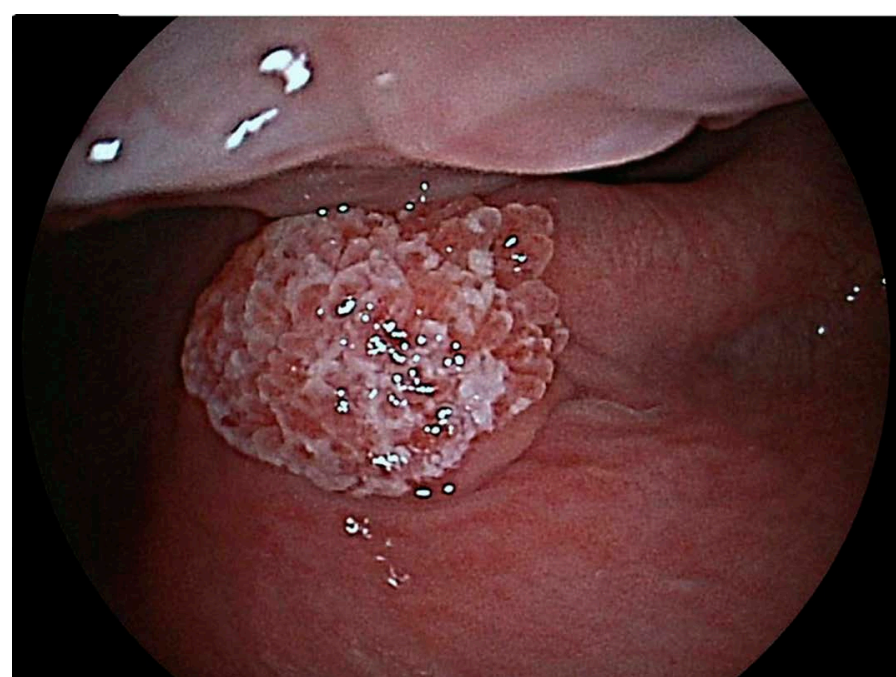

Figure 4. The endoscopic view of another primary cancer located at pharyngoesophageal junction using the trumpet maneuver.

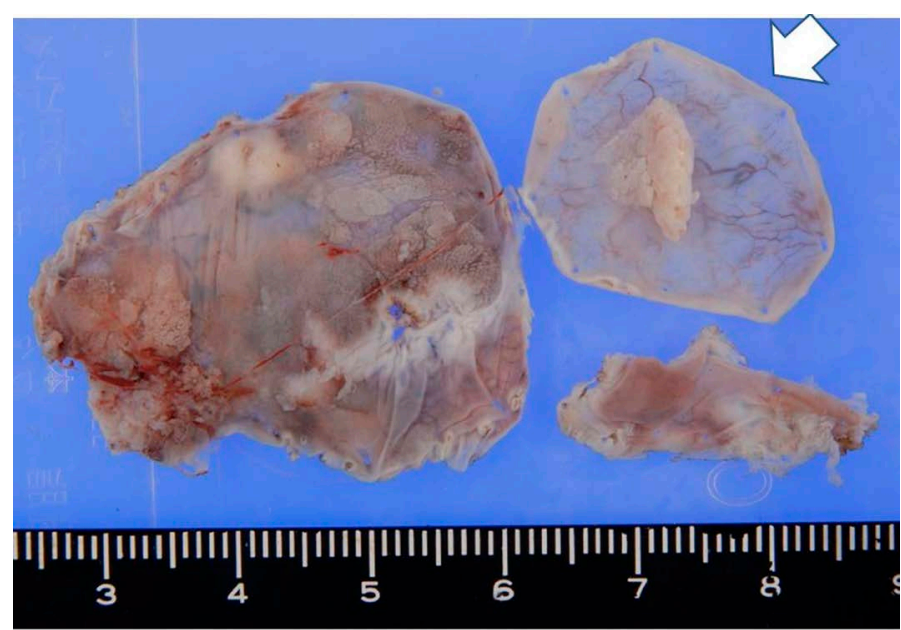

Figure 5. Macroscopic appearance of the resected specimens (arrows).

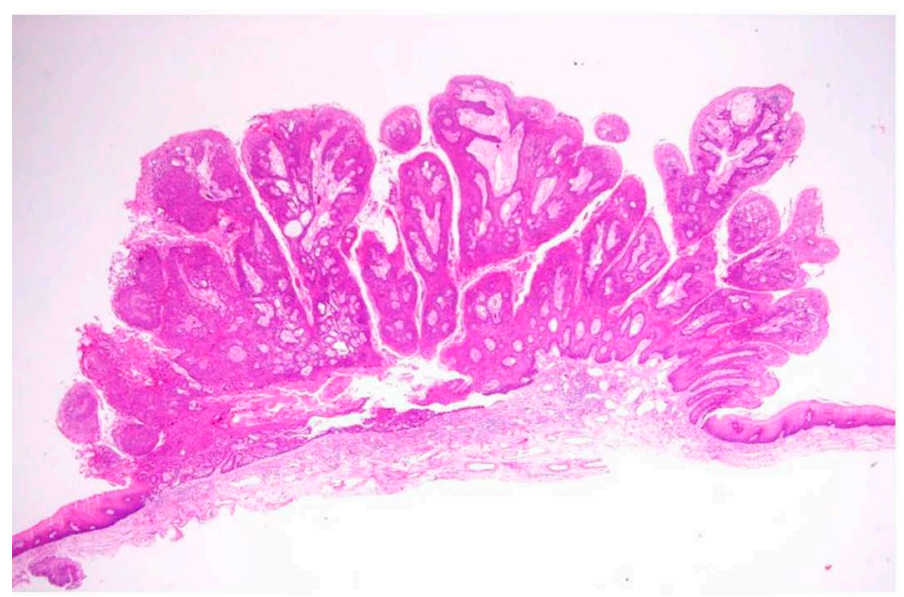

Figure 6. Histopathological examination revealed a diagnosis of invasive squamous cell carcinoma. 
need for conventional iodine staining for the diagnosis of squamous cell carcinoma [2]-[4]. A rhinolaryngoscope equipped with an NBI system is also available for examining these lesions. Watanabe et al. [5] [6] reported that image enhanced rhinolaryngoscopy improves the diagnostic accuracy, sensitivity, and negative predictive value for detecting squamous cell carcinoma of the head and neck (SCCHN) in patients with esophageal cancer. Esophagogastroduodenoscopy (EGD) has also been improved, but some patients cannot be examined at otorhinolaryngeal sites due to their gag reflex. The postcricoid subsite and the orifice of the esophagus are especially difficult to visualize using flexible laryngopharyngoscopy.

Several reports have suggested techniques for improving the view of the hypopharynx with a flexible fiberoptic laryngoscope. Spraggs and Harris described a modified Valsalva technique involving the nose being squeezed shut by the examiner's hand while the patient attempted to blow through the obstructed nose [7] [8]. Other reports have described the trumpet maneuver [9], the anterior neck skin traction maneuver [10] or a combination of the two [11]. However, this maneuver has not been attempted for conventional EGD because of the gag reflex.

Transnasal esophagogastroduodenoscopy (EGD) can be performed comfortably because of its attenuation of the gag reflex. However, there are some disadvantages to this procedure compared with conventional endoscopy, such as a decrease in the brightness, image resolution and suction ability, so the diagnostic abilities of this system have not been sufficiently evaluated. However, the procedure has recently been making tremendous progress, and a new endoscope (model EG-580NW, FUJI Film, Tokyo, Japan) was manufactured beginning in October of 2011. The new endoscope is a trans-nasal endoscope that provides high quality endoscopic images of a wide field view (of $140^{\circ}$ ). On conventional EGD, we usually insert the endoscope from the left pyriform sinus of the hypopharynx to the cervical esophagus, and when we pull out the endoscope, it passes the left or right side of the pyriform sinus making the postcricoid subsite, the posterior of the hypopharynx, and the orifice of the esophagus difficult to visualize on conventional EGD.

In this case, we performed the trumpet maneuver with trans-nasal endoscopy. Adhesion of the posterior wall and the post-cricoid subsite was lifted completely, and then another primary lesion was observed at the posterior wall of the hypopharynx near the pharyngoesophageal junction.

The procedure for wide visualization of the pharynx provided an excellent endoscopic field of view of pharynx which is not possible using conventional EGD and was useful to detect the very early superficial type of head and neck cancers. It is well known that multiple squamous cell carcinomas frequently arise in the upper aerodigestive tract either synchronously or metachronously [12]. As a result, the value of performing endoscopic screening for high risk patients has been emphasized in order to detect second malignancies at an earlier stage.

\section{Conclusion}

In conclusion, the trumpet maneuver using trans-nasal endoscopy is a useful technique for precise inspection before treatments for patients who have already detected the lesions.

\section{References}

[1] Shah, J.P., Shaha, A.R., Spiro, R.H., et al. (1976) Carcinoma of the Hypopharynx. The American Journal of Surgical Pathology, 132, 439-443. http://dx.doi.org/10.1016/0002-9610(76)90315-9

[2] Muto, M., Nakane, M., Katada, C., et al. (2004) Squamous Cell Carcinoma in Situ at Oropharyngeal and Hypopharyngeal Mucosal Sites. Cancer, 101, 1375-1381. http://dx.doi.org/10.1002/cncr.20482

[3] Muto, M., Katada, C., Sano, Y., et al. (2005) Narrow Band Imaging: A New Diagnostic Approach to Visualize Angiogenesis in Superficial Neoplasia. Clinical Gastroenterology and Hepatology, 3, 16-20. http://dx.doi.org/10.1016/S1542-3565(05)00262-4

[4] Muto, M., Minashi, K., Yano, T., et al. (2010) Early Detection of Superficial Squamous Cell Carcinoma in the Head and Neck Region and Esophagus by Narrow Band Imaging: A Multicenter Randomized Controlled Trial. Journal of Clinical Oncology, 28, 1566-1572. http://dx.doi.org/10.1200/JCO.2009.25.4680

[5] Watanabe, A., Tsujie, H., Taniguchi, M., et al. (2006) Laryngoscopic Detection of Pharyngeal Carcinoma in Situ with Narrowband Imaging. The Laryngoscope, 116, 650-654. http://dx.doi.org/10.1097/01.mlg.0000204304.38797.34

[6] Watanabe, A., Taniguchi, M., Tsujie, H., et al. (2008) The Value of Narrow Band Imaging Endoscope for Early Head and Neck Cancers. Otolaryngology-Head and Neck Surgery, 138, 446-451. http://dx.doi.org/10.1016/j.otohns.2007.12.034

[7] Spraggs, P.D. and Harries, M.L. (1995) The Modified Valsalva Maneuver to Improve Visualization of the Hypopharynx during Flexible Nasopharyngoscopy. Journal of Laryngology and Otology, 109, 863-864. 
http://dx.doi.org/10.1017/S0022215100131512

[8] Purser, S. and Antippa, P. (1995) Maneuver to Assist Examination of the Hypopharynx. Head Neck, 17, $389-393$. http://dx.doi.org/10.1002/hed.2880170505

[9] Hillel, A.D. and Schwartz, A.N. (1989) Trumpet Maneuver for Visualization and CT Examination of the Pyriform Sinus and Retrocricoid Area. Head Neck, 11, 231-236. http://dx.doi.org/10.1002/hed.2880110307

[10] Colquhoun-Flannery, W., Davis, A. and Carruth, J.A.S. (2000) Improving the Endoscopic View of the Hypopharynx with Anterior Neck Traction during the Trumpet Manoeuvre. Journal of Laryngology and Otology, 114, $283-284$. http://dx.doi.org/10.1258/0022215001905562

[11] Williams, R.S., Lancaster, J., Karagama, Y., et al. (2004) A Systematic Approach to the Nasendoscopic Examination of the Larynx and Pharynx. Clinical Otolaryngology \& Allied Sciences, 29, 175-178. http://dx.doi.org/10.1111/j.0307-7772.2004.00784.x

[12] Morita, M., Kuwano, H., Ohno, S., et al. (1994) Multiple Occurrence of Carcinoma in the Upper Aerodigestive Tract Associated with Esophageal Cancer: Reference to Smoking, Drinking and Family History. International Journal of Cancer, 58, 207-210. http://dx.doi.org/10.1002/ijc.2910580211

\section{Video}

The transnasal endoscope was inserted to the pharynx. The primary pharyngeal cancer was detected easily. There was no another tumor from left pyriform sinus to the cervical esophagus. Among the trumpet maneuver, the pharyngeal cancer located at the posterior wall of the hypopharynx near the pharyngoesophageal junction was detected. We could get the biopsy specimen easily using transnasal endoscopy. 
Scientific Research Publishing (SCIRP) is one of the largest Open Access journal publishers. It is currently publishing more than 200 open access, online, peer-reviewed journals covering a wide range of academic disciplines. SCIRP serves the worldwide academic communities and contributes to the progress and application of science with its publication.

Other selected journals from SCIRP are listed as below. Submit your manuscript to us via either submit@scirp.org or Online Submission Portal.
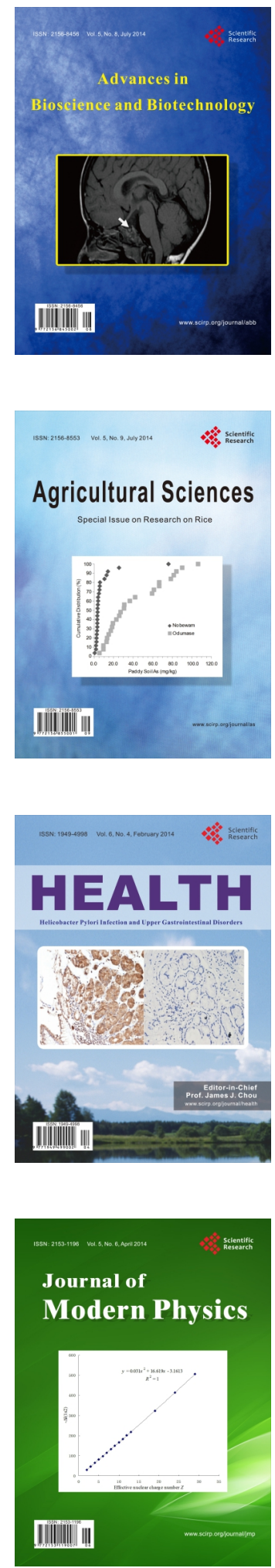
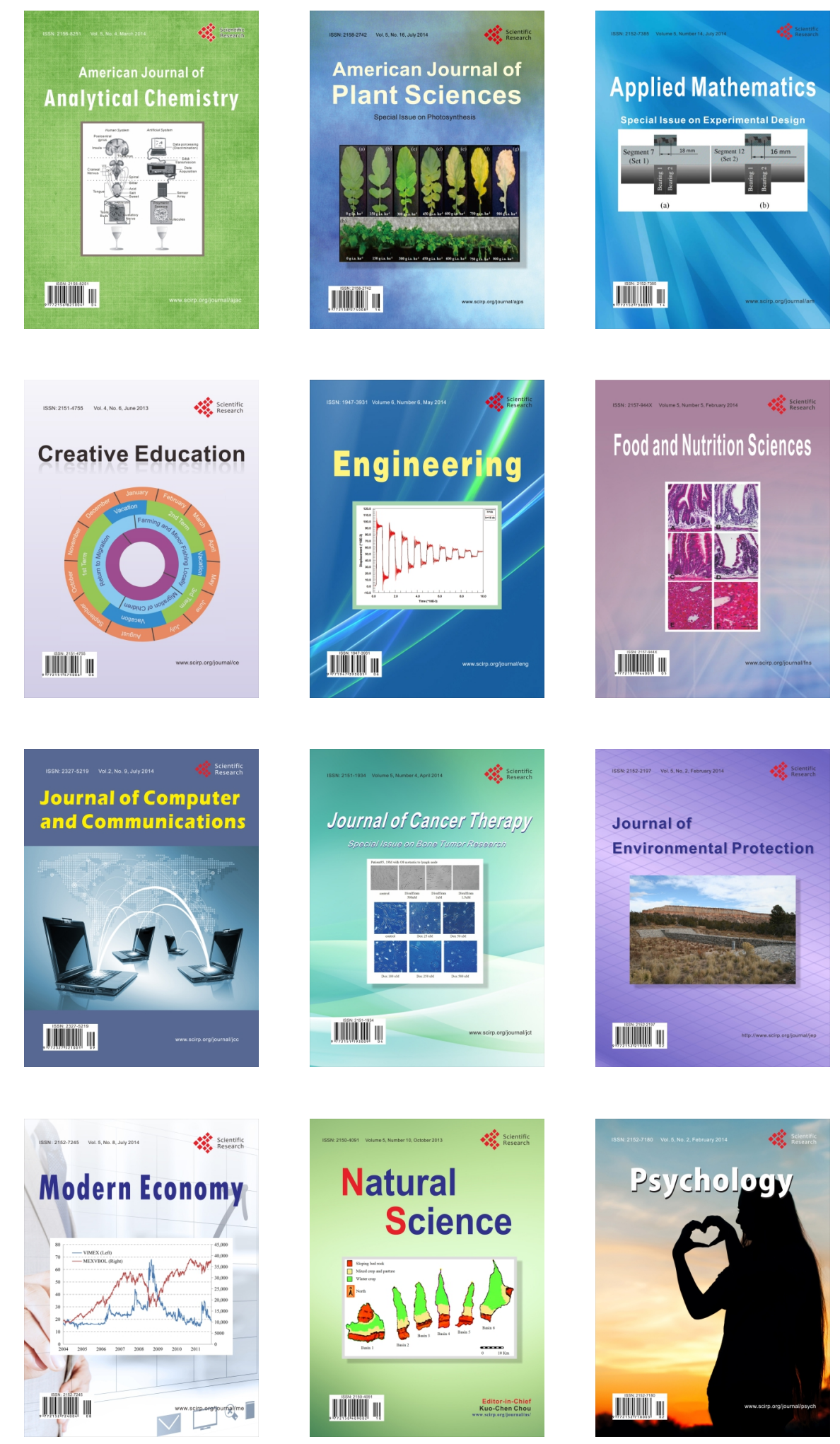